\title{
Thermal conductivity of quasi-one-dimensional antiferromagnetic spin-chain materials
}

\author{
A. V. Rozhkov ${ }^{1}$ and A. L. Chernyshev ${ }^{1}$ \\ ${ }^{1}$ Department of Physics, University of California, Irvine, California 92697
}

(Dated: November 12, 2018)

\begin{abstract}
We study heat transport in quasi-one-dimensional spin-chain systems by considering the model of one-dimensional bosonic spin excitations interacting with three-dimensional phonons and impurities in the limit of weak spin-lattice coupling and fast spin excitations. A combined effect of the phonon and impurity scatterings yields the following spin-boson thermal conductivity behavior: $\kappa_{s} \propto T^{2}$ at low, $\kappa_{s} \propto T^{-1}$ at intermediate, and $\kappa_{s}=$ const at higher temperatures. Our results agree well with the existing experimental data for $\mathrm{Sr}_{2} \mathrm{CuO}_{3}$. We predict an unusual dependence on the impurity concentration for a number of observables and propose further experiments.

PACS numbers: 75.10.Pq, 71.10.Pm, 72.10.Bg, 75.40.Gb
\end{abstract}

Introduction. Recent experiments in one-dimensional (1D) quantum magnets have revealed remarkably strong thermal transport anomalies associated with the lowdimensional spin degrees of freedom. In particular, an anisotropic thermal conductivity, comparable in magnitude to that of metallic systems, was observed in quasi-1D spin- $\frac{1}{2}$ chain and ladder compounds with a large part of the heat current attributed to magnetic excitations. 1, 2] Only recently, however, a considerable progress in the theoretical understanding of the thermal transport properties of $1 \mathrm{D}$ quantum spin systems has been made. 3 ]

In this Letter we consider the heat transport in spinchain systems within the Boltzmann equation framework. We develop a microscopic approach to the problem of 1D spin-boson excitations interacting with the 3D phonon environment and impurities. An experimental realization of such a system is $\mathrm{Sr}_{2} \mathrm{CuO}_{3}$. 1]

Generally, in order to study transport problems one needs to identify a mechanism responsible for the momentum relaxation. Conventional examples are the Umklapp and impurity scatterings. Since the characteristic "bandwidth" for the spin excitations in the real 1D spin chains materials of interest is very large $(J \sim 2000 \mathrm{~K})$ in comparison with the experimental temperature range, the Umklapp scattering of spin excitations on themselves is strongly suppressed (as $\sim e^{-J / T}$ ). Instead, we propose that the leading relaxation mechanism at not too low temperatures is the two-stage, bottle-neck process of (i) transferring the momentum from spins to phonons, and (ii) the subsequent dissipation of the momentum via a phonon Umklapp process. The central idea is that the stage ( $i$ ) is the bottle-neck because the spin-lattice coupling is very weak. Thus, the excess momentum in the spin subsystem waits the longest time to get transfered to phonons, but once it is transferred it relaxes quickly. If this is the case we have to find only the spin-phonon relaxation time $\tau_{s p}$ that now plays the role of transport relaxation time for the spin excitations. However, as we demonstrate below, the spin-phonon scattering mechanism alone becomes too weak for the low-lying excitations which leads to an infinite spin thermal conductivity $\kappa_{s}$. On the other hand, the impurity scattering is very strong in $1 \mathrm{D}$ at low energies. This scattering mechanism renders $\kappa_{s}$ finite for any realistic system and allows us to provide a detailed description of the thermal transport.

Model. The Hamiltonian of a single Heisenberg antiferromagnetic spin- $\frac{1}{2}$ chain is:

$$
H_{\text {chain }}=J \sum_{i} \mathbf{S}_{i} \cdot \mathbf{S}_{i+1} .
$$

The interchain coupling is very small in $\left.\mathrm{Sr}_{2} \mathrm{CuO}_{3}, 1\right]$ $J^{\prime} / J \sim 10^{-5}$, and is neglected here. After the JordanWigner transformation followed by bosonization Eq. (1) can be expressed as the Hamiltonian of free bosonic field $\Phi$, which we refer to as the spin-boson field:

$$
H_{0}=\frac{v}{2} \int d x\left(\Pi^{2}+\left(\partial_{x} \Phi\right)^{2}\right)=v \sum_{k}|k| b_{k}^{\dagger} b_{k},
$$

where $\Pi(x)$ is a conjugate field momentum, and velocity $v$ is given by $v \propto J a, a$ is the chain lattice constant, [5] and $b, b^{\dagger}$ are the spin-boson operators.

Since the superexchange $J$ is a function of the inter-site distance the lattice vibrations can modify it. This will couple phonons to the spin-boson Hamiltonian density. Such an interaction can be written in terms of the phonon operators $a, a^{\dagger}$ and the spin-boson operators as follows:

$$
\begin{gathered}
H_{\mathrm{sp}}=-\frac{g_{s p}}{\sqrt{N}} \sum_{k k^{\prime} \mathbf{P} \ell} V_{\ell}\left(\mathbf{P}, k, k^{\prime}\right)\left(a_{\mathbf{P} \ell}^{\dagger} b_{k}^{\dagger} b_{k^{\prime}}+\text { h.c. }\right) \\
V_{\ell}\left(\mathbf{P}, k, k^{\prime}\right)=\frac{P_{\|} k k^{\prime}}{\sqrt{8 m_{i} \omega_{\mathbf{P} \ell} k k^{\prime}}}\left(\boldsymbol{\xi}_{\mathbf{P} \ell}\right)_{x} \delta_{P_{\|}, k^{\prime}-k},
\end{gathered}
$$

where $g_{s p}$ is a spin-phonon coupling constant, the index $\ell$ enumerates three phonon polarizations, $\omega_{\mathbf{P} \ell}=c_{\ell}|\mathbf{P}|$ is the phonon dispersion, $\boldsymbol{\xi}$ is the polarization vector of a phonon, $m_{i}$ is the mass of the unit cell, $N$ is the number of unit cells in the sample, $k$ and $k^{\prime}$ are the 1D momenta of spin bosons, and $\mathbf{P}$ is the $3 \mathrm{D}$ momentum of a phonon. Note that only a component of the total momentum along the chain is conserved, which is explicitly given by the $\delta$ function. Sound velocities $c_{\ell}$ are much smaller than the 
spin-boson velocity $v$ for the real spin-chain materials: $c_{\ell} \ll v$. Eq. (3) describes the processes in which spin boson either emits or absorbs a phonon. The projection of the phonon polarization vector on the direction of the chain ( $x$-axis) $\left(\boldsymbol{\xi}_{\mathbf{P} \ell}\right)_{x}$ is equal to: $\left|P_{\|}\right| /|\mathbf{P}|$ (longitudinal), and $\sqrt{1-\left(P_{\|} / \mathbf{P}\right)^{2}}$ or 0 (transverse), where two answers for the transverse phonons correspond to the polarization in the plane given by $\hat{x}$ and $\mathbf{P}$ and normal to such a plane, respectively. The latter phonons do not couple to spins and are not discussed any further.

The impurity coupling is the final component of the model. Impurities lead to a local variation of the superexchange $J$, that leads to the scattering of magnetic excitations. The most important (relevant in the renormalization group (RG) sense) part of the impurity Hamiltonian for an impurity located at $x_{0}$ can be written as:

$$
H_{\mathrm{imp}}=\frac{\delta J_{\mathrm{imp}}}{\pi} \cos \left(2 k_{\mathrm{F}} x_{0}+\sqrt{4 \pi \mathcal{K}} \Phi\left(x_{0}\right)\right),
$$

where the Luttinger-liquid parameter $\mathcal{K}=1 / 2$.

Phonon mechanism of relaxation. To study the relaxation of 1D spin excitations coupled to the bath of 3D phonons we solve the stationary Boltzmann equation: $v \partial_{x} f_{k}=-S_{k}[f]$, where $f_{k}$ is the spin-boson distribution function and $S_{k}[f]$ is the collision integral, given by:

$$
S_{k}[f]=\int_{k^{\prime}}\left[W_{k k^{\prime}} f_{k}\left(f_{k^{\prime}}+1\right)-W_{k^{\prime} k} f_{k^{\prime}}\left(f_{k}+1\right)\right],
$$

where $\int_{k^{\prime}}$ stands for $\int d k^{\prime} / 2 \pi$ and $W_{k k^{\prime}}$ is the total probability of the spin excitation to be scattered from the state $k$ to the state $k^{\prime}$. For the processes of scattering due to phonons such probabilities are given by:

$$
W_{k k^{\prime}}=\sum_{\ell} \int_{\mathbf{P}}\left(w_{k k^{\prime} \mathbf{P}}^{\ell}\left(n_{\mathbf{P} \ell}^{0}+1\right)+w_{k^{\prime} k \mathbf{P}}^{\ell} n_{\mathbf{P} \ell}^{0}\right),
$$

where $\int_{\mathbf{P}}$ denotes $(1 / 2 \pi)^{3} \int d^{3} \mathbf{P}$. The probability $W_{k^{\prime} k} \equiv$ $W_{k k^{\prime}}\left[k \leftrightarrow k^{\prime}\right]$. We assume that for all relevant temperatures the phonon relaxation time is much shorter than that of the spin bosons. Consequently, one can use the equilibrium form for the phonon distribution function $n_{\mathbf{P} \ell}=n_{\mathbf{P} \ell}^{0}=1 /\left(e^{\omega_{\mathbf{P} \ell} / T}-1\right)$. The "elementary" scattering probabilities $w_{k k^{\prime} \mathbf{P}}^{\ell}$ of the spin boson due to emission or absorption of the phonon with the momentum $\mathbf{P}$ and polarization $\ell$ are determined from Eqs. (3), (4):

$$
\begin{aligned}
w_{k k^{\prime} \mathbf{P}}^{\ell}= & \frac{g_{s p}^{2} V_{0}}{8 m_{i}} \cdot \frac{P_{\|}^{2}\left|k k^{\prime}\right|}{\omega_{\mathbf{P} \ell}}\left(\boldsymbol{\xi}_{\ell}\right)_{x}^{2} \\
& \times \delta\left(k^{\prime}+P_{\|}-k\right) \delta\left(\omega_{k^{\prime}}+\omega_{\mathbf{P} l}-\omega_{k}\right),
\end{aligned}
$$

where $V_{0}$ is the elementary cells volume, $\omega_{k}=v|k|$ is the spin-boson energy. Note that the total energy and the projection of the momentum along the chain are conserved, which is enforced by the $\delta$-functions in Eq. (8).

For a small temperature gradient $\partial_{x} T \neq 0$ one can introduce a usual ansatz for the distribution function $f_{k}$ to linearize the Boltzmann equation: $f_{k}=f_{k}^{0}+f_{k}^{1}$, where $f_{k}^{1}$ is a non-equilibrium correction to the equilibrium Bose distribution function $f_{k}^{0}$. Function $f_{k}^{1}$ should be small and proportional to the temperature gradient. Thus, the Boltzmann equation for the spin bosons to the first order in $\partial_{x} T$ can be written as:

$$
\frac{v|k|}{T}\left(\partial_{x} T\right) \frac{\partial f_{k}^{0}}{\partial k}=S_{k},
$$

where $S_{k}$ is the linearized collision integral given by $S_{k}=$ $\sum_{\ell}\left(S_{k \ell}^{(1)}+S_{k \ell}^{(2)}\right)$ with

$$
S_{k \ell}^{(1)}=\int_{k^{\prime} \mathbf{P}} w_{k k^{\prime} \mathbf{P}}^{\ell}\left[P_{k, \mathbf{P}}^{(1)} f_{k}^{1}+P_{k, \mathbf{P}}^{(2)} f_{k^{\prime}}^{1}\right],
$$

where $P_{k^{\prime}, \mathbf{P}}^{(1)}=\left(n_{\mathbf{P} \ell}^{0}+f_{k^{\prime}}^{0}+1\right), P_{k, \mathbf{P}}^{(2)}=\left(f_{k}^{0}-n_{\mathbf{P} \ell}^{0}\right)$ and $S_{k \ell}^{(2)} \equiv-S_{k \ell}^{(1)}\left[k \leftrightarrow k^{\prime}\right]$. The integral $S^{(1)}$ accounts for two types of collision events: (i) spin boson with the momentum $k$ emits a phonon with the momentum $\mathbf{P}$ and scatters into the state with the momentum $k^{\prime}$; (ii) process inverse to (i). Likewise, $S^{(2)}$ describes absorption of a phonon by the spin boson with the momentum $k$ and the corresponding inverse process.

Once $f_{k}^{1}$ is found the spin-boson thermal current density $J_{E}^{s}$ can be written as: $J_{E}^{s}=\int_{k} v^{2} k f_{k}^{1}=-\kappa_{s} \partial_{x} T$, where the coefficient $\kappa_{s}$ is the spin-boson thermal conductivity. Note that the use of Boltzmann equation implies the semiclassical transport, condition for which can be shown to be satisfied for the real 1D spin system.

Kinematic considerations. To solve the Boltzmann equation one needs to evaluate integrals in Eq. (10). Part of this task can be accomplished without any approximations as the integral over the phonon momentum $\mathbf{P}$ in $S^{(1)}$ and $S^{(2)}$ can be calculated explicitly. The remaining integration over $k^{\prime}$ is restricted to some finite intervals due to the conservation of the energy and momentum. [4]

Further, there is a following simplification. As we noted above, without impurities the heat conductivity will diverge at small $|k|$. Thus, the biggest contribution to $\kappa_{s}$ comes from the low-energy spin bosons: $v|k| \ll \min \left(T, \Theta_{D}\right)$ and we need to consider only them.

Relaxation time. One can write the collision integral in Eq. (10) as a sum of two terms: $S_{k}=f_{k}^{1} / \tau_{s p}(k)+\delta S_{k}$, where the first term has the usual relaxation time form, while the second one does not. However, the second term can be demonstrated to be small for small spin-boson energy $\delta S_{k} / S_{k} \propto(v k / T)^{2}$.

Finally, with the help of this approximation, the collision integral can be written in the relaxation time form $S_{k} \approx f_{k}^{1} / \tau_{\mathrm{sp}}(k)$, where $\tau_{\mathrm{sp}}^{-1}(k)$ plays the role of the transport relaxation rate and is given by: [4]

$$
\tau_{\mathrm{sp}}(k)^{-1}=\frac{\mathcal{A} k^{2} T^{3}}{v^{3}} \Gamma\left(\Theta_{D} / T\right),
$$

where $\Gamma(z)=\frac{I_{1}(z)}{I_{1}(\infty)}$, and $I_{1}(z)=\int_{0}^{z} \frac{x^{3} d x}{2 \sinh ^{2} x / 2}$. Here the phenomenological constant $\mathcal{A}$ is of the order of 
$\left(V_{0} / m_{i}\right)\left(g_{s p} / c\right)^{2}$. By evaluating the asymptotic properties of $\Gamma(z)$ one can easily verify that:

$$
\tau_{\mathrm{sp}}(k)^{-1}= \begin{cases}\mathcal{A} T^{3} k^{2} / v^{3} & \text { for } T<\widetilde{\Theta}_{D}, \\ \mathcal{A} \widetilde{\Theta}_{D}^{2} T k^{2} / v^{3} & \text { for } T>\widetilde{\Theta}_{D},\end{cases}
$$

where $\widetilde{\Theta}_{D}=\Theta_{D} / \sqrt{I_{1}(\infty)} \approx \Theta_{D} / 4$ plays the role of a crossover temperature. The relaxation rate for the spin boson, Eq. (12), is our main result for the spin-phonon scattering. We note that the $k$ - and $T$-dependence of the bottle-neck relaxation rate will be the same for other low-D spin systems with large $J$ where momentum is conserved in the dimensions fewer than $\mathrm{D}=3$.

It is interesting to note that since the spin bosons are fast $(c \ll v)$, the energy and momentum conservation dictates that the majority of the phonons which interact with the spin subsystem must have their momentum almost normal to the chain direction. Indeed, the phonon energy is $\omega_{\mathbf{P}}=c P=v|| k|-| k^{\prime}||$, where $k$ and $k^{\prime}$ are the 1D spin-boson momenta, while the momentum conservation along the chain gives $\left|P_{\|}\right|=\left|k-k^{\prime}\right|$. Therefore, for a typical phonon: $P \gg\left|P_{\|}\right|$, except for the case of almost elastic backward scattering $\left(k^{\prime} \approx-k\right)$ which does not contribute substantially to the transport relaxation time. Consequently, we determine $\boldsymbol{\xi}_{x l}=\mathcal{O}(c / v)$ and $\boldsymbol{\xi}_{x t}=\mathcal{O}(1)$. Thus, the most effective spin-phonon scattering is due to the transverse phonons.

With the collision integral in the relaxation time form the Boltzmann equation is trivially solved:

$$
f_{k}^{1}=\frac{v|k| \tau_{\mathrm{sp}}(k)}{T}\left(\partial_{x} T\right) \frac{\partial f_{k}^{0}}{\partial k} \approx-\frac{\tau_{\mathrm{sp}}(k)}{k}\left(\partial_{x} T\right),
$$

and the thermal conductivity is calculated:

$$
\kappa_{s}=\int v^{2} \tau_{\mathrm{sp}}(k) \frac{d k}{2 \pi}=\frac{v^{5}}{2 \pi \mathcal{A} T^{3}} \int_{0}^{T / v} \frac{d k}{k^{2}} .
$$

This expression diverges at small $k$ giving rise to an infinite thermal conductivity. It occurs because the scattering of spin bosons on phonons is not sufficiently strong for $k \rightarrow 0$ to ensure the convergence of the low-energy contribution to the integral.

Impurities. In order to remove the divergence of (14) we need to introduce yet another scattering mechanism for the low- $k$ spin bosons. In a 1D system impurities scatter low-energy excitations very effectively. Therefore, disorder in the magnetic coupling $J$ will regularize the infrared divergence in Eq. (14). Since the spin-impurity interaction in Eq. (5) is not a low-order polynomial in $\Phi$, the Boltzmann transport theory cannot be directly applied to the impurity scattering. Instead, we will evaluate the spin boson life-time $\tau_{\text {imp }}$ using the Green's function perturbative expansion in powers of $\delta J_{\mathrm{imp}}$.

There are two issues we must clarify before proceeding with this approach. First, for the perturbation theory in powers of $\delta J_{\text {imp }}$ to be valid the temperature must be bigger than the Kane-Fisher temperature: $[\underline{6}] T \gg T_{K F}$. For our system with $\mathcal{K}=1 / 2$ the Kane-Fisher temperature is $T_{\mathrm{KF}}=\delta J_{\mathrm{imp}}^{2} / J$. Second, it is important to note that, generally, the life-time of an excitation is not equivalent to the transport relaxation time. While the only processes which violate the conservation of the momentum contribute to the latter, any kind of scattering shortens the former. However, since Eq. (5) does not conserve momentum and since in any typical scattering event the spin-boson momentum changes drastically, there is no distinction between these two time scales in our case. This justifies the use of our approach.

The second-order correction to the spin-boson Green's function from the impurity scattering is:

$$
\delta \mathcal{D}_{k}^{(2)}(\tau)=\int_{\tau^{\prime} \tau^{\prime \prime}}\left\langle B_{k}^{\dagger}(\tau) \overline{H_{\mathrm{imp}}\left(\tau^{\prime}\right) H_{\mathrm{imp}}\left(\tau^{\prime \prime}\right)} B_{k^{\prime}}(0)\right\rangle,
$$

where $B_{k}^{\dagger}(\tau)=b_{k}^{\dagger}(\tau)+b_{-k}(\tau)$ and the line over the Hamiltonians stands for the disorder averaging. The right hand side of this equation is proportional to the Matsubara self-energy. After analytical continuation we find the retarded self-energy: [4]

$$
\Sigma_{k, \omega}^{R} \approx-i \frac{n \delta J_{\mathrm{imp}}^{2} \omega}{a \pi^{2} J|k| T},
$$

with $n$ being the dimensionless impurity concentration. Once the self-energy is found one can obtain the dressed Green's function: $D_{k, \omega}=2 \omega_{k} /\left(\omega_{k}^{2}-\omega^{2}-2 \omega_{k} \Sigma_{k, \omega}^{R}\right)$. By finding the poles of this expression the damping of the spin bosons is determined as given by:

$$
\tau_{\mathrm{imp}}^{-1}=\frac{\Delta^{2}}{T},
$$

where $\Delta^{2} \propto n \delta J_{\text {imp }}^{2}$. [7] Unlike in the spin-phonon case the impurity relaxation rate is independent of $k$ and thus will remove the divergence of the thermal conductivity.

Thermal conductivity. Finally, having at hands the transport relaxation rates for the spin boson due to phonon and impurity scattering, Eqs. (11) and (16), we can calculate the thermal conductivity of the spin chains. The total relaxation time can be found according to the Matthiessen's rule: $\tau_{\text {tot }}{ }^{-1}=\tau_{\mathrm{imp}}{ }^{-1}+\tau_{\mathrm{sp}}{ }^{-1}$. Then the expression for the thermal conductivity (14) should be modified to:

$$
\kappa_{s}(T)=\frac{v T^{2}}{\pi \Delta^{2}} \int_{0}^{J / T} \frac{x^{2} d x}{4 \sinh ^{2}(x / 2)} \frac{\alpha(T)}{x^{2}+\alpha(T)},
$$

with $\alpha(T)=v^{5} \Delta^{2} / \mathcal{A} T^{6} \Gamma\left(\Theta_{D} / T\right)$. Since the temperature is always much smaller than $J$ one can safely replace the upper limit in Eq. (17) by infinity.

Eq. (17) specifies a function with a single maximum at $T=T_{m} \propto\left(n \delta J_{\text {imp }}^{2} / \mathcal{A} J\right)^{1 / 6}$, the maximum value of $\kappa_{s}$ is $\kappa_{s}^{\max }=\kappa_{s}\left(T_{m}\right) \propto v^{6} / \mathcal{A} T_{m}^{4}$. The function $\kappa_{s}(T)$ vanishes as $T^{2}$ at $T \ll T_{m}$ and saturates at $T \gg T_{m}$, approaching 


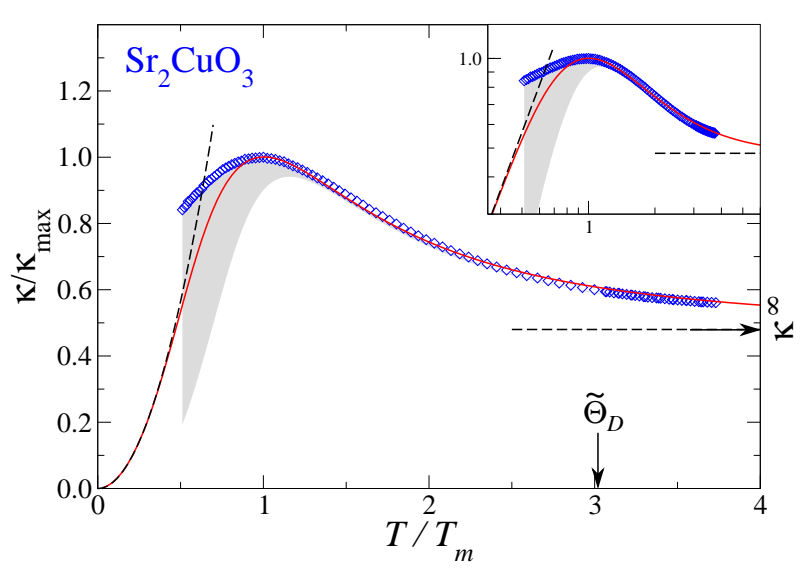

FIG. 1: Normalized spin thermal conductivity $\kappa_{s}$ vs reduced temperature $T / T_{m}$. Diamonds are the experimental data for $\mathrm{Sr}_{2} \mathrm{CuO}_{3}, T_{m}=79.4 \mathrm{~K}$ and $\kappa_{s}^{\max }=36.7 \mathrm{Wm}^{-1} \mathrm{~K}^{-1}$, see Ref. 1. Solid line is the result of this work, Eq. (17), for $\Theta_{D}=$ $11.6 T_{m}$. Arrows mark the saturation value $\kappa_{s}^{\infty}$ for the solid line at $T \gg \widetilde{\Theta}_{D}$ and the spin-phonon scattering crossover scale $\widetilde{\Theta}_{D}$, respectively. Asymptotic behavior at $T \ll T_{m}(\sim$ $\left.T^{2}\right)$ and at $T \gg T_{m}(\sim$ const $)$ are shown by the dashed lines. Inset: same data on the log-log scale.

$$
\begin{aligned}
& \kappa_{s}^{\infty} \propto \kappa_{s}^{\max } T_{m} / \Theta_{D}: \\
& \quad \frac{\kappa_{s}(T)}{\kappa_{s}^{\max }} \approx \begin{cases}2.26 T^{2} / T_{m}^{2} & \text { for } T \ll T_{m}, \\
1.46 T_{m} / T & \text { for } T_{m} \ll T \ll \widetilde{\Theta}_{D}, \\
5.55 T_{m} / \Theta_{D} & \text { for } T \gg \widetilde{\Theta}_{D} .\end{cases}
\end{aligned}
$$

At temperatures below $T_{m}, \kappa_{s}$ is controlled by impurity scattering. 8] At temperature $T>T_{m}$ the number of higher energy spin bosons, which scatter mostly on phonons, increases. However, the low- $k$ spin bosons are still scattered mostly by impurities. Thus, at $T \gg T_{m}$ both mechanisms contribute to $\kappa_{s}$.

Comparison with experiments. Using Eq. (17) we compare our theory with the experimental data for $\mathrm{Sr}_{2} \mathrm{CuO}_{3}$. It is convenient to measure the temperature in units of $T_{m}$ and the thermal conductivity in units of $\kappa_{s}^{\max }$. This guarantees that the location of the maximum coincides with the maximum of the experimental curve. Once this rescaling is done there is only one parameter to adjust in our theory: $\Theta_{D}$. By choosing $\Theta_{D}=11.6 T_{m}$ we fit the experimental data for $T>T_{m}$ almost ideally. Shaded area at $T<T_{m}$ highlights the region where the phonon background subtraction creates a large uncertainty in the experimental data. 1] With this in mind we conclude that Eq. (17) describes very well the whole range of available experimental data.

Our work predicts a remarkable behavior of the thermal conductivity at higher temperatures: saturation at a constant value. A parallel can be drawn with metals, where $\kappa$ also saturates at high temperatures. However, in our case the excitations are not fermions at $E_{F}$, but the long-wavelength spin bosons. Because of that, the manner in which impurity scattering cuts off such exci- tations at low energies remains important even at high temperatures. Therefore, in the case of spin chains the saturation of $\kappa_{s}$ is a result of a non-trivial combination of several effects: temperature- and $k$-dependence of the impurity and phonon relaxation rates and the $1 \mathrm{D}$ density of states of the spin-bosons. We note that the saturated regime in $\kappa_{s}$ should persist up to $T \sim J$ above which bosonic description of excitations is not adequate.

Predictions. One of the most straightforward ways to verify our theory would be to measure the thermal conductivity for higher temperatures and check if the saturation really takes place as we predict. Next, we see that the temperature $T_{m}$ at which $\kappa_{s}(T)$ reaches its maximum scales as $n^{1 / 6}$. Also, the maximum value of the thermal conductivity $\kappa_{s}^{\max }=\kappa_{s}\left(T_{m}\right)$ scales as $n^{-2 / 3}$, an unusual and rather strong effect. Finally, the saturation value $\kappa_{s}^{\infty}$ has yet different concentration dependence $n^{-1 / 2}$. If such a behavior is observed in the materials with the isotope substitution for either $\mathrm{Cu}$ or $\mathrm{O}$ ions it will provide a strong support to our theory.

Conclusions. To conclude, we have developed a theory of anomalous heat transport in the spin-chain systems coupled to the $3 \mathrm{D}$ phonon environment in the presence of weak disorder. We have calculated the thermal conductivity as a function of temperature and have obtained that the low-temperature transport is dominated by the impurity scattering while the high temperature transport is determined by both the impurity scattering and the spin-phonon collisions. Our main results are in a very good quantitative agreement with the available experimental data. In implementing our approach we have also obtained an insight into various microscopic details of the problem. This has allowed us to formulate several predictions and suggest future experiments.

This work was supported by DOE under grant DEFG02-04ER46174.

[1] A.V. Sologubenko et al., Phys. Rev. B 64, 054412 (2001).

[2] A.V. Sologubenko et al., Phys. Rev. Lett. 84, 2714 (2000); Europhys. Lett. 62, 540 (2003); C. Hess et al., Phys. Rev. B 64, 184305 (2001); Phys. Rev. Lett. 90, 197002 (2003).

[3] X. Zotos, Phys. Rev. Lett. 82, 1764 (1999); J. V. Alvarez and C. Gros, ibid., 88, 077203 (2002); F. Heidrich-Meisner et al., Phys. Rev. B 66, R140406 (2002); K. Saito ibid., 67, 064410 (2003); E. Orignac et al., ibid., 67, 134426 (2003); E. Shimshoni et al., ibid., 68, 104401 (2003).

[4] For the technical details see cond-mat/0407257

[5] A. O. Gogolin et al., Bosonization and Strongly Correlated Systems, (Cambridge University Press, 1998).

[6] C. L. Kane and M. P. A. Fisher, Phis. Rev. B 46, 15233 (1992).

[7] A similar expression has been found in M. Oshikawa and I. Affleck, Phys. Rev. B 65, 134410 (2002).

[8] same $\kappa \sim T^{2}$ has been obtained in M. R. Li and E. Orignac, Europhys. Lett. 60, 432 (2002). 